\title{
Towards Electron-Beam-Driven Soft / Polymer Fiber Microrobotics for Vacuum Conditions
}

\author{
Oleg V. Gradov ${ }^{1, a^{*}}$, Margaret A. Gradova1,b, Irina A. Maklakova ${ }^{1,2, c}$ and \\ Svetlana N. Kholuiskaya ${ }^{1, d}$ \\ 1Semenov Federal Research Center for Chemical Physics of the Russian Academy of Sciences, \\ 119991, Kosygin Street 4, Moscow, Russia \\ ${ }^{2}$ Institute of Biochemical Physics named after N.M. Emanuel of the Russian Academy of \\ Sciences, 119991, Kosygin Street 4, Moscow, Russia \\ ao.v.gradov@gmail.com, bm.a.gradova@gmail.com, c maklakosha@yandex.ru, \\ ds_n_khol@mail.ru
}

Keywords: Polymer Fibers, Electron Beam, Microrobotics, Vacuum Microrobotics, Elastic Cantilever, Polymer Fiber Spring, Electron Beam Driven MEMS, Electron Beam Control, 2D Fourier Spectra, Integral Spatial Characteristics, Time Resolved Correlation, Real Time Correlation-Spectral Analysis

\begin{abstract}
The possibility of creating vacuum robotics based on the polymer structures irradiated by an electron beam, in particular, polymer fibers, which provide high functional flexibility and a variety of states, is discussed. The possibility of using polymer fibers as different types of MEMSlike electromechanical elements is demonstrated - from elastic cantilevers to springs that change their state under the electron beam. Experimentally proved the presence of different functional types of fibers, correlating with their thickness, as well as the phenomenon of the fiber break. A number of exotic forms of dynamics have been demonstrated and a method for their detection has been developed using 2D Fourier spectra, integral spatial characteristics, time resolved correlograms and wavelet transforms (visualized as the scaleograms / scalograms). Access barcodes for the full video records of the corresponding experiments are provided.
\end{abstract}

\section{Introduction}

Vacuum, ultra-high vacuum and cryovacuum microrobotics are at the frontiers of the modern mechatronics development. Design of the robot machine for ITER (International Thermonuclear Experimental Reactor) vacuum vessel assembly and maintenance assumes that the robot can operate in vacuum vessel conditions [1-6]. Recently, similar work is underway in China aimed at the design of a fusion reactor CFETR (Chinese Fusion Engineering Test Reactor [7]). The authors of the last cited work [7] emphasize that "the assembly of vacuum vessel (VV) is a vital task for the fusion reactor" and any violation of the technological conditions during the VV assembly or operation is an emergency situation. A similar situation is observed in microelectronics, where the robots for use in ultrahigh vacuum are also useful for wafer-transfer manipulations [8-12], and the disruption of the processing modes leads to multimillion-dollar losses from any defective batch of the mass chips. Accordingly, the controllability of robots in vacuum conditions is of high importance. In the case of vacuum robots for microelectronics, the most widely used are magnetic transmission technology or coaxial twin-shaft magnetic fluid seals applied in vacuum waferhandling robots [13]. Operation stability at the macroscales is achieved by controlling the magnetic field since magnetic switching for microrobotics is a much more difficult task. The problem of position-sensitive control in vacuum microrobotics is still poorly developed. 
At the same time, for microrobotic tasks that do not allow working with cables (in contrast to macroscales as in a cable-driven robotized arm for tracking in TOKAMAK vacuum vessels [14]), the size of which may exceed the cross-sections of the actuated structures, it is advisable to create conditions for the contactless signal transduction at the scales from the order of hundreds of nanometers up to several microns.

For a "cable" approach application, another method of solving the above problem is possible combining a vacuum "microcable" and an extended micromanipulator in a single structure.

However, the question of supplying a signal to the input unit (sensor and actuator) remains open. In this case, it is possible to propose the use of the antenna properties of the cable to receive the input signal, especially for transmission of the signal to the cable using a focused microbeam corresponding in size at the projection point to the size of the antenna and the actuated structure. The use of radio frequency signals for actuation at these scales is not possible, since the "antenna" dimensions are much smaller than the wavelength (excluding the terahertz range, where the physics of metamaterials operates).

Therefore, it is proposed to use the excitation of "antennas" integrated with the "microcables" for signal transduction and fibrillar or flexible actuators (powered by the energy of the signal supplied to the antenna), by the focused electron microbeam of the medium energy that does not disrupt the structure, but provides kinematics of its movement. Application of metal wire structures for these purposes is not suitable both due to the metallographic reasons and their fractographic consequences, and due to the high densities of the beam energy flux, which must be supported to the material in order to ensure its deformation and movement of the metal wires (this task is more close to the electron beam melting than to the problem of the material deformation under the electron beam).

Therefore, perhaps the only rational approach is the use of polymer / fibrillar-composite materials, the extended components of which can serve both as an antenna and an actuator, and also possess simple cable or membrane-mimetic properties, which ensure the signal transduction along the surface / "membrane" of the actuators [15]. It is noteworthy that despite the vacuum robotics history starting from the last quarter of the XX century [16-18], the above approach integrating all functions in a single structure in accordance with TRIZ requirements, has not been found in the literature. This indicates the novelty and promising nature of the approach proposed and opens the prospects of its wide practical application.

\section{Materials and Methods}

Polyhydroxybutyrate (PHB) 16F was purchased from BIOMER Company and used as received. PHB molding solutions with a PHB concentration of $7 \mathrm{wt} \%$ in chloroform were used to obtain ultra-thin fibers by electrospinning (ESP) method.

Position-sensitive registration of the dielectric charging process and fiber dynamics was carried out using scanning electron microscope JEOL JSM-T330A at the accelerating voltage of $10 \mathrm{kV}$. In order to observe the dielectric charging phenomena no metal sputtering has been applied on the sample surface before the SEM studies.

Photo- and video registration of the experiments was performed using a system developed by P. Alexandrov (Shemyakin-Ovchinnikov Institute of Bioorganic Chemistry of the Russian Academy of Sciences) and O. Gradov. Access codes for the full video records of all the experiments in Code-128 standard are given at the bottom of the corresponding tables and figures.

2D Fourier spectra and integral spatial characteristics (ISC) were obtained in real time using QAVIS software based on the FFTW library, developed in V.I. Il'ichev Pacific Okeanological Institute (Far Eastern Branch of the Russian Academy of Sciences). 
Charge distribution mapping for "integrated antenna and cable" structures was performed using a discrete differentiation operator, computing an approximation of the gradient of the image intensity function - isotropic 3x3 image gradient operator, or Sobel - Feldman operator (from SAIL - Stanford Artificial Intelligence Laboratory).

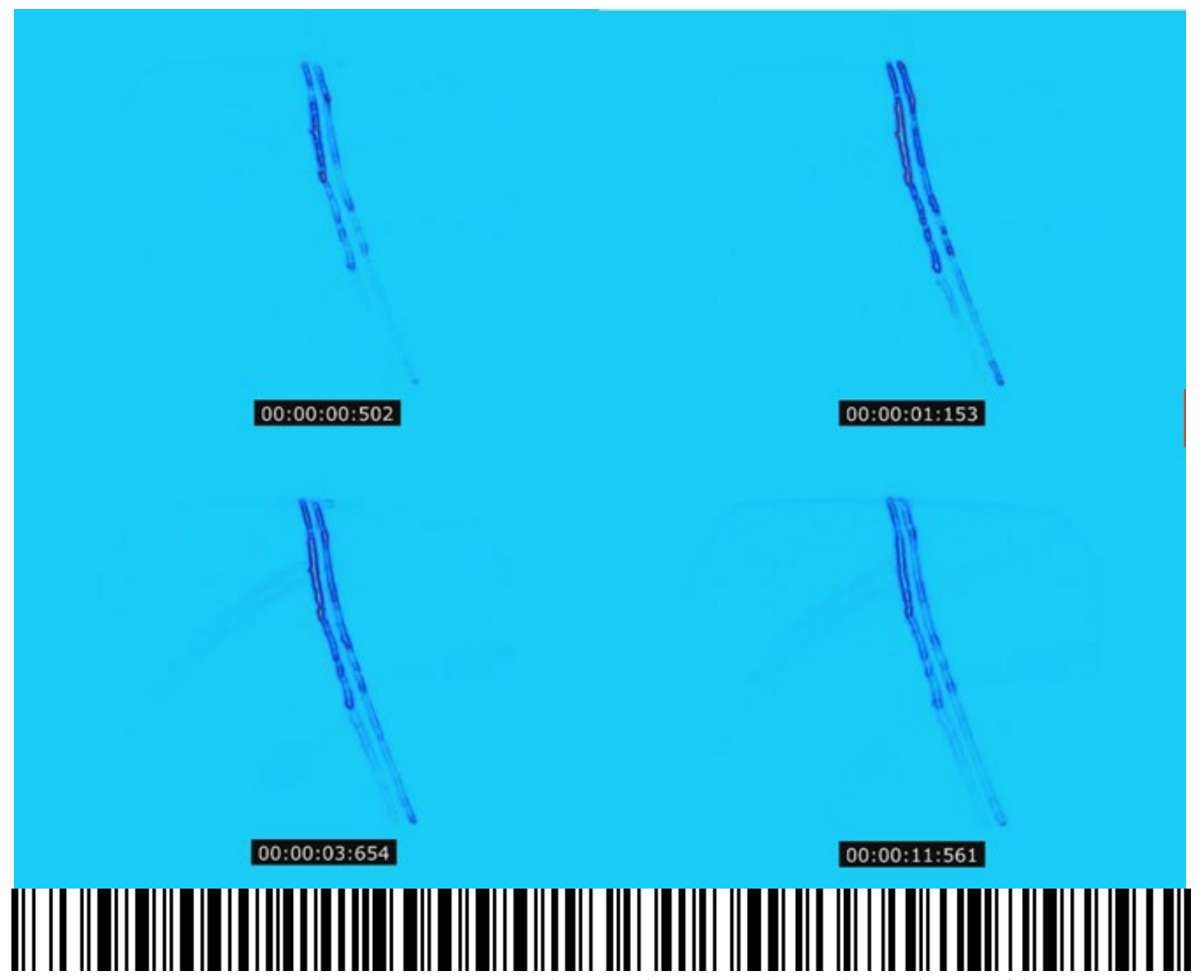

Fig. 1. Signal "receiving" and transduction along the polymer fiber.

(Full video recording of this experiment is available at https://youtu.belepWhGKAzCtE).

\section{Results}

Initially we studied the ability of a non-sputtered PHB fiber to "receive" an electron beam signal, which was estimated by the fact of dielectric charging of the single fiber surface (see Fig. 1). The possibility of the discrete signal propagation (i.e., charge distribution) over the dielectric surface and the presence of attenuation phenomena were also observed. The contribution of the surface inhomogeneities to maintaining a discrete structure of the charge distribution was also demonstrated.

After evaluating the ability of a non-sputtered microfiber to "receive" an electron beam signal, we studied the micromechanics of its dynamics and response to the changes in the operation modes of the electron microscope (including the electron beam focusing, magnification, accelerating voltage, etc.).

As you can see from the table 1, after increasing the power density, polymer fibers begin to move rapidly under the electron beam and "relax to a metastable state" in a little more than half a minute. Up to this point, a complete reconfiguration of the fiber directions occurs, depending on 
their position under the beam, as can be seen from the comparison of 2D Fourier spectra and Integral Spatial Characteristics (ISC) at different times. After that, reversible fiber oscillations also occur, but the whole network topology remains stable, which is consistent with the stability of the ISC symmetry axes, the angular ratios of which vary by only a few degrees.

Table 1.

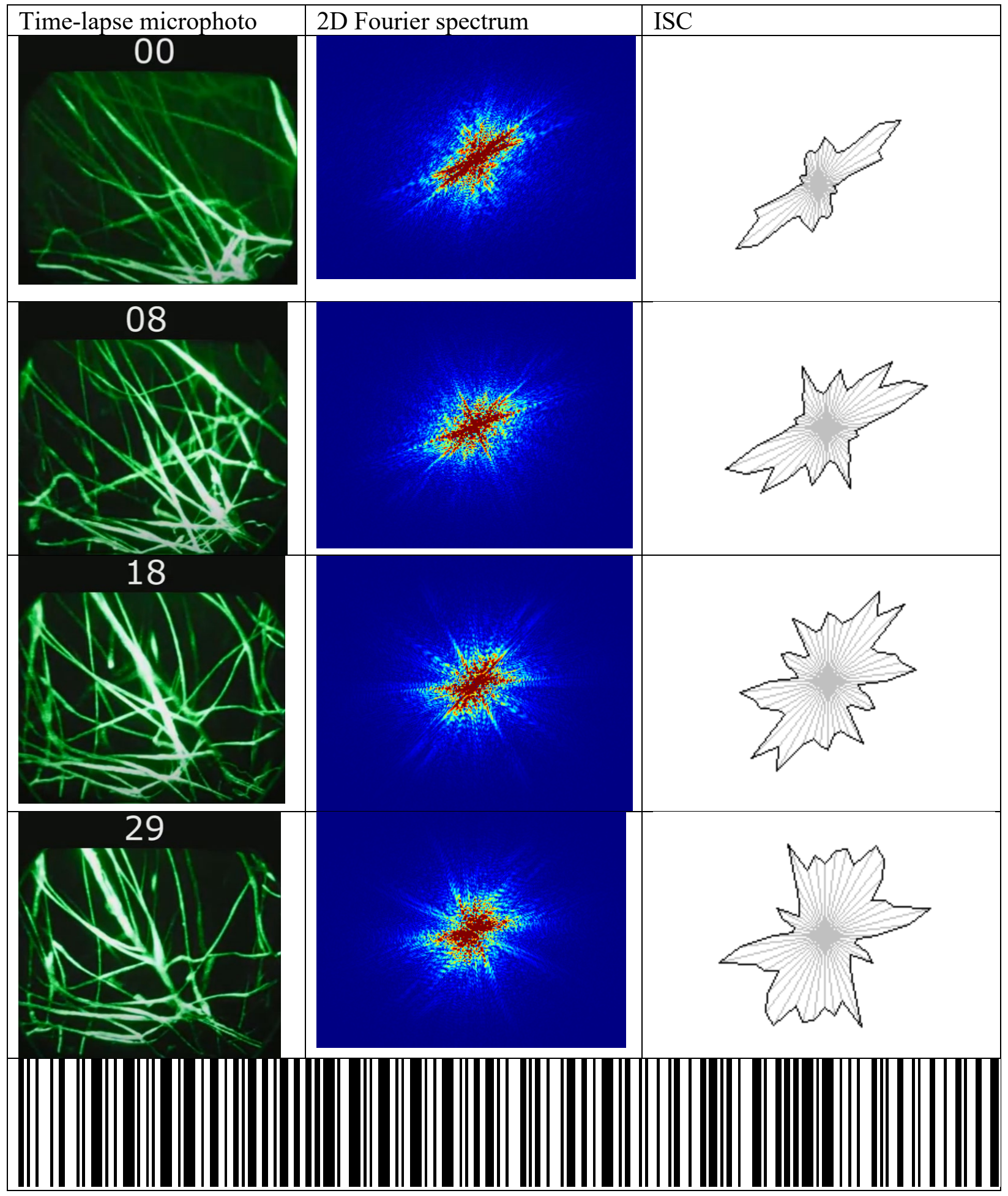

Video recording of the experiment is available at https://youtu.be/uHBLeWysbAA. 
In some cases, not smooth movements are observed, leading to a change in the network configuration under the electron beam, but spontaneous instantaneous changes accompanied by a sharp increase or disappearance of ISC components, as shown in Table. 2.

Table 2.

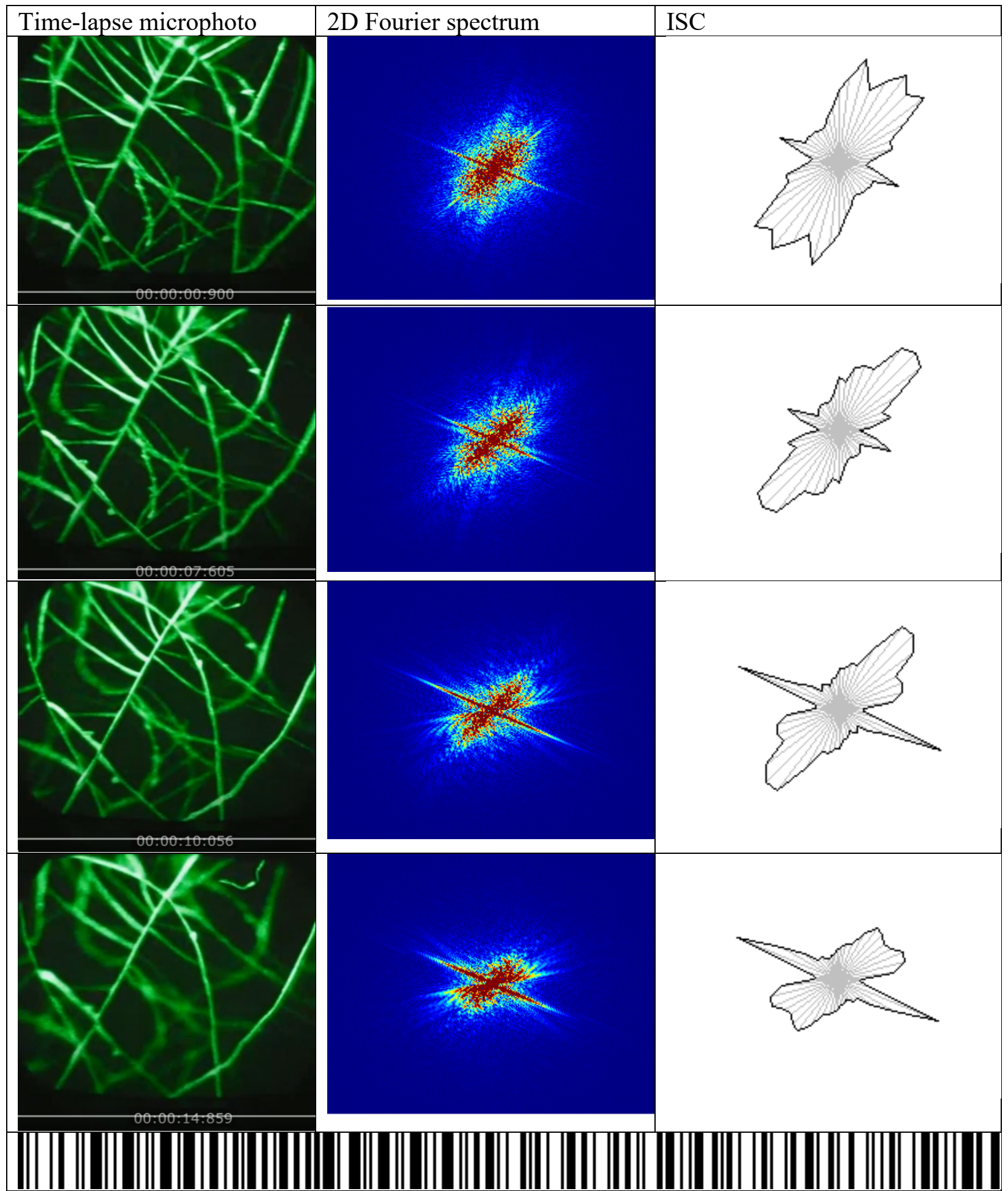

Video recording of the experiment is available at https://youtu.be/cl5NegKEng0 . 
Table 3.

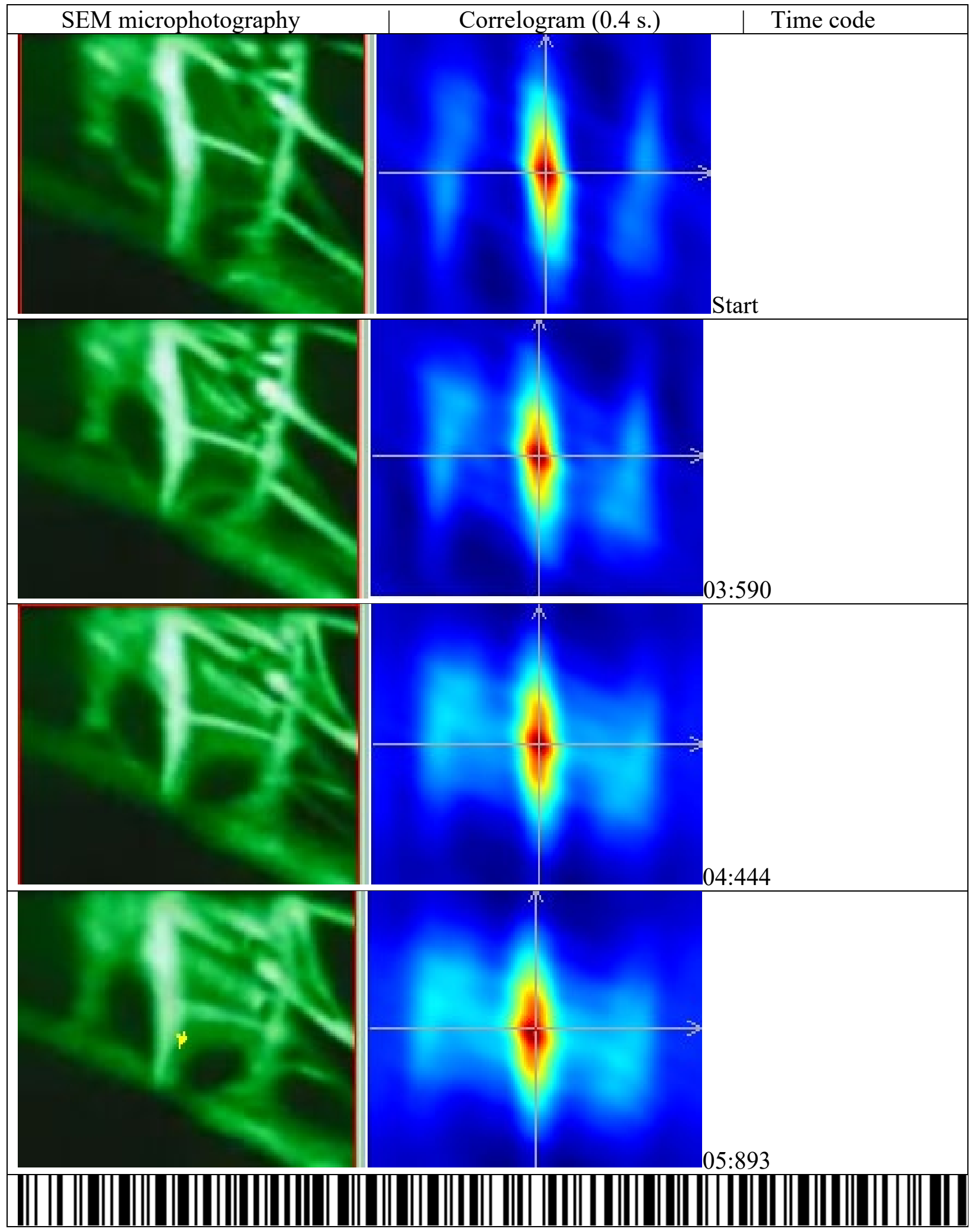

Video recording of the experiment is available at https://youtu.be/A9tVG6Zu2sI . 
In some cases, a reorganization of the entire structures is observed, which can be applied for the electron-beam-controlled microrobotics. Thus, table 3 includes micrographs and correlograms taken with a temporal resolution of 0.4 seconds for such a reorganized area. It is obvious that exposure to an electron beam induces mechanical changes in the cross-linked micromechanical structure of the polymer. In this case, different fibers work as "jacks", "drive belts", "springs", "elastic beams", "pushers", etc. The mechanical effect observed depends on the irradiated area, that is, the effective cross-sectional area of the scanning electron beam.

It is possible to confirm the assumption that some (thick) fibers perform the functions of bearing elements of mechanical strength, while the others (thin) operate as the flexible elements controlled by the electron beam. Let us consider the experiment with two different structures. In table 4 one can see the movement of a thin flexible fiber (the thread in the upper part of the micrograph) against the stability of the large thick one (in the center). The fiber movement features can be tracked using the data of the wavelet transform (Morlet wavelet) in the Re and Im regions (one can observe the drift of the extremum on the "blue" representation).

The measurements were carried out using the scalographic / scaleographic tool of the QAVIS program (see http://oias.poi.dvo.ru/qavis/rus/scalogram.html), which makes it possible to use the appropriate mathematical formalization for their interpretation, excluding the ineffective Gaussian approach.

If some fibers oscillate, move, contract like springs (thin fibers), while others perform a frame function (thick fibers), then the micromechanical properties of the polymer fibers should be assessed in dynamics. It is obvious that a fiber break is a possible cause of damage for the polymerbased controlled microelectromechanical structures, including those for microrobotic or MEMS applications. Therefore, mechanical strength tests should be performed for each chemical or structural type of polymer fibers under the electron beam with different parameters. An example of a PHB fiber break clearly observed on the SEM image is shown in Fig. 2.

\section{Discussion}

Where can the above described concept be applied? Obviously, its implementation is possible in different systems operating under vacuum conditions with the priority application area being space technologies. Polymer materials in vacuum are used in dozens of space applications, including vacuum-based 3D printing for in-space satellite assemblies [19]. Possible damages of polymer materials in outer space, including the effect of cosmic rays and elementary particles, are well known and predictable [20-24]. Therefore, the development of polymer robotics for space technologies is not a problem from modern materials science. From the point of view of mechanics, it is necessary to simulate vibrations in a vacuum without taking into account the earth's gravitational force [25], but this is rather a simplification than a complication of the problem.

Table 4. 


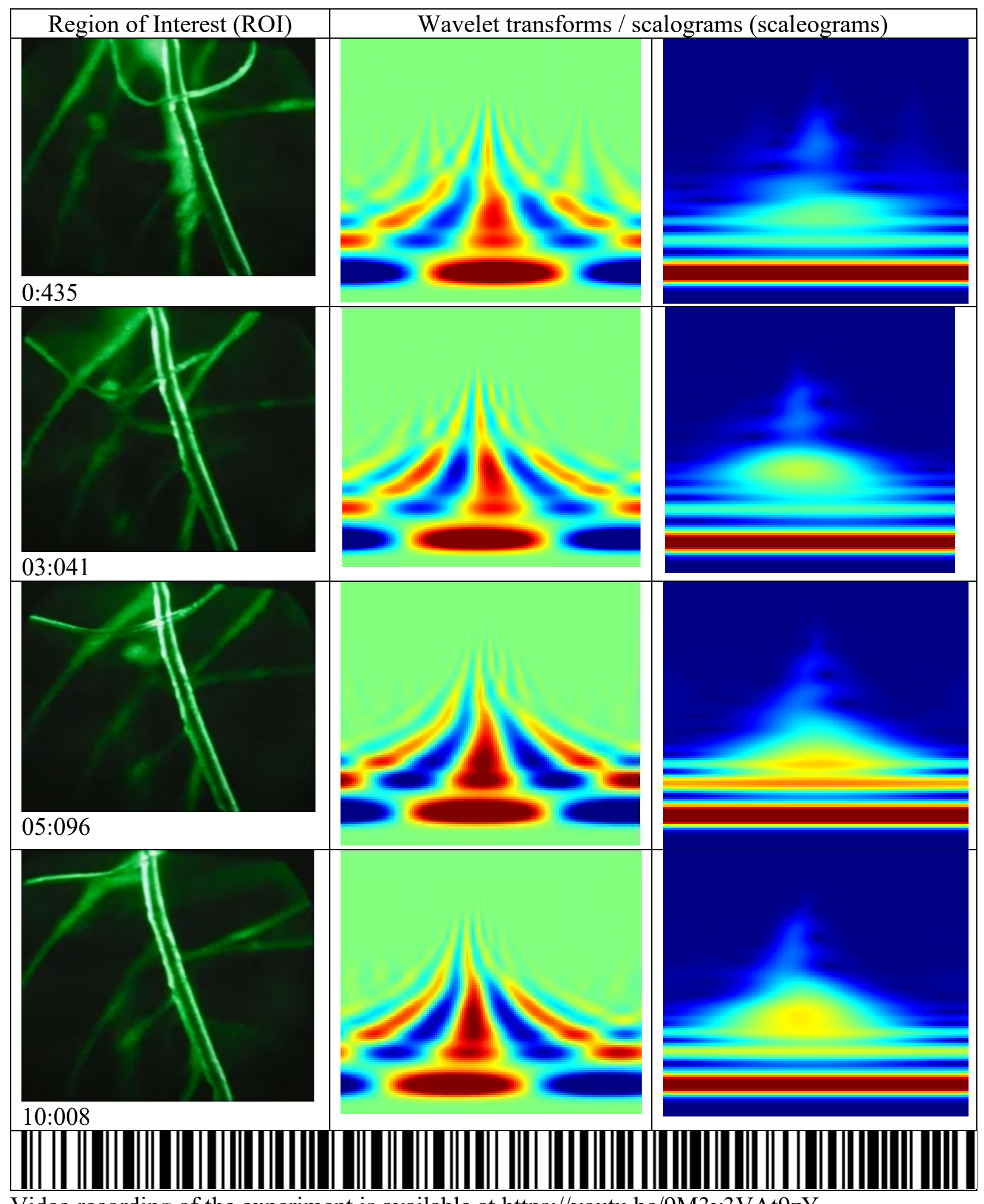

Video recording of the experiment is available at https://youtu.be/9M3y3VAt9zY 


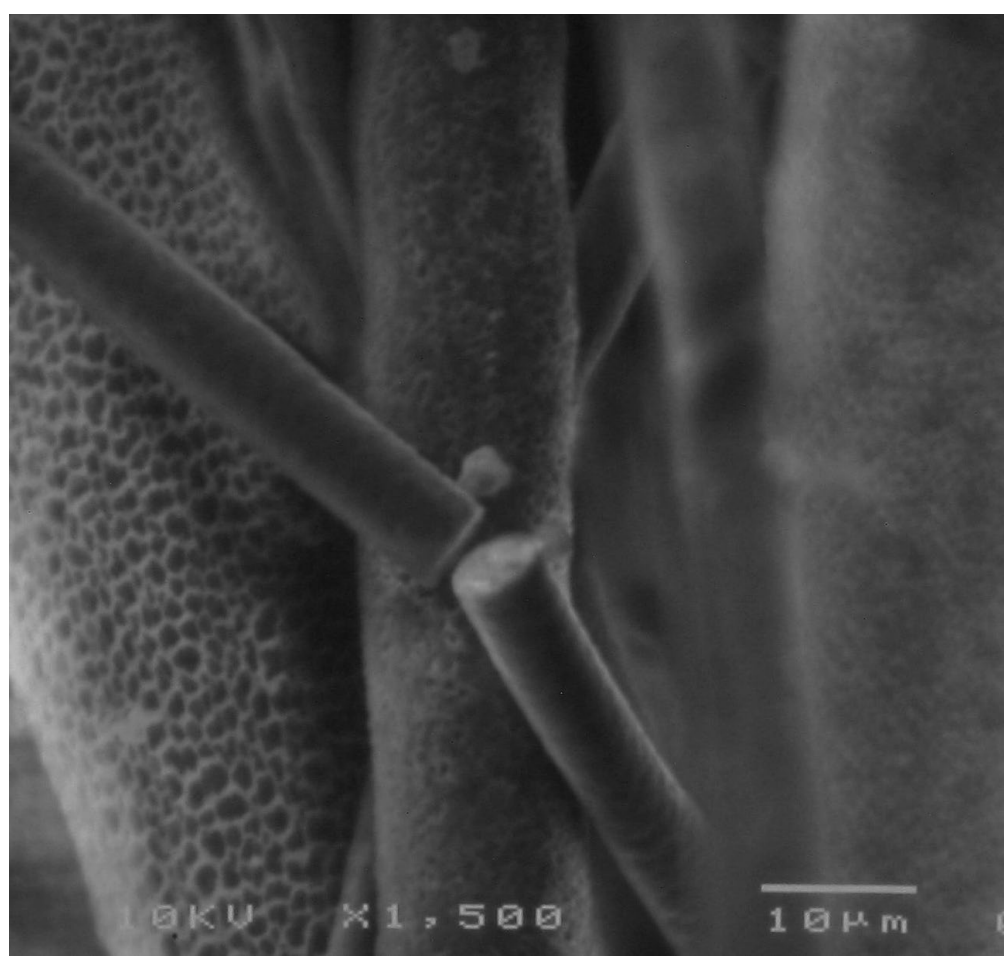

Fig. 2. Example of the broken polymer fiber.

Speaking about the application of robotics in space technologies, it is necessary to take into account its long history - from the late 1960s (sensu lato, taking into account the automated Babakin spacecrafts in the USSR and a number of similar developments in the USA) or, at least, since the mid-1970s (sensu stricto, considering robotic manipulators with automatic decision making function) for interstellar research [26,27]. The main fundamental modern requirements for interstellar robotics are formulated in departmental international meetings of the late 20th - early 21 st centuries $[28,29]$. The difference between the extended interpretation of space robotics and its narrow interpretation is that both mechanically active systems for sensing the external environment and passive mapping systems [30,31] are included in the first, while the narrow interpretation includes only active mechatronic elements such as robotic arms $\backslash$ manipulators [32].

In our concept, a narrow interpretation prevails, since the dynamics of microfibers, cantilevers under the particle beam makes sense only if there are moving elements - actuators in the spacecraft construction. A similar interpretation is also typical for environment - robot interactions for planetary or terrestrial subsurface explorers / robots based on earthworm locomotion $[33,34]$ and hopping robots for planetary or asteroid surface exploration [35-37]. It should be noted that asteroids do not have an atmosphere and sufficient gravitational force, while planets usually have it. Therefore, in the first case, we can speak about the vacuum systems, which are essentially equivalent to the experimental vacuum conditions in SEM and TEM, and in the second case, only about the similarity with ESEM (Environmental Scanning Electron Microscopy) and ASEM (Atmospheric Scanning Electron Microscopy).

We are not considering here aerial robot explorers for planetary exploration [38], which by definition are not vacuum devices. For the same reason robots with manipulators for exploring planets are beyond the scope of this paper [39-42]. Therefore, in this case, we can only talk about the applications in "open space" and non-atmospheric bodies: meteorites and others [43]. As 
practice shows, this indirect and not always accessible way of solving problems (the transition from macroscale to microscale robotics and the introduction of non-standard interpretations despite the inconsistency with the high-budget mainstream) can help in obtaining non-standard results using spacecraft, as was the case decades ago [44-46]. A fibrillar manipulator cannot replace a macromanipulator, but for nanosatellites and picosatellites (such as "CanSat"), sets of cooperative fibrillar manipulators with the electron beam control may be the only possible means of achieving efficiency in many mechanical applications including pulsed one-time manipulations (such as opening a mirror or a solar battery) [47-57].

Launching CanSat into orbit is a separate technical problem, since the suborbital trajectories do not meet the requirements for sufficient evacuation degree and weightlessness. That is, we can speak about the applicability in open space beyond the Karman line $(>100 \mathrm{~km})$, and not beyond the Armstrong physiological line of $19 \mathrm{~km}$. However, vacuum studies of potentially applicable polymers have not yet been carried at all the necessary gradations of heights and characteristics of the medium (from atmospheric to vacuum ones). Thus, one can only postulate the presence of a kinematic effect on polymer fibers under the electron beam and speculate about its potential applicability for the purposes of space technology. It should be noted that not all the polymers used in our studies are resistant to the conditions of outer space during long-term exposure. Therefore, this material can be considered only as the first step in this direction, illustrating the physical effect, but not specifying its chemical implementation.

\section{Acknowledgements}

The authors are grateful to V.K. Fischenko and A.A. Goncharova from the V.I. Il'ichev Pacific Okeanological Institute of Far Eastern Branch of the Russian Academy of Sciences for providing the original software. We also gratefully acknowledge M. K. Filippov and P. L. Aleksandrov for automation of the electron microscope, and A.V. Ratnovskaya for assistance in SEM image and video registration.

\section{References}

[1] H. Wu, H. Handroos, J. Kovanen, A. Rouvinen, P. Hannukainen, T. Saira, L. Jones, Design of parallel intersector weld/cut robot for machining processes in ITER vacuum vessel, Fusion Engineering and Design 69(1-4) (2003) 327-331. https://doi.org/10.1016/S0920-3796(03)00066-8

[2] H. Wu, H. Handroos, P. Pessi, Mobile parallel robot for assembly and repair of ITER vacuum vessel, Industrial Robot 35(2) (2008) 160-168. https://doi.org/10.1108/01439910810854656

[3] J.C. Hatchressian, V. Bruno, L. Gargiulo, D. Keller, Y. Perrot, P. Bayetti, J.J. Cordier, J.P. Friconneau, J.D. Palmer, F. Samaille. Development of an inspection robot under ITER relevant vacuum and temperature conditions, Journal of Physics: Conference Series 100(6) (2008) 062031. https://doi.org/10.1088/1742-6596/100/6/062031

[4] M. Li, H. Wu, H. Handroos, G. Yang, Software design of the hybrid robot machine for ITER vacuum vessel assembly and maintenance, Fusion Engineering and Design 88(9-10) (2013) 1872 1876. https://doi.org/10.1016/j.fusengdes.2013.04.023

[5] H. Wu, Y. Wang, M. Li, M. Al-Saedi, H. Handroos, Chatter suppression methods of a robot machine for ITER vacuum vessel assembly and maintenance, Fusion Engineering and Design 89(9-10) (2014) 2357-2362. https://doi.org/10.1016/j.fusengdes.2014.02.007

[6] M. Li, H. Wu, H. Handroos, G. Yang, Y. Wang, Software protocol design: Communication and control in a multi-task robot machine for ITER vacuum vessel assembly and 
maintenance, Fusion Engineering and Design 98 (2015) 1532-1537.

https://doi.org/10.1016/j.fusengdes.2015.05.058

[7] S. Moradkhani, Y.S. Hagh, H. Wu, H. Handroos, Dynamic analysis and control of a fusion reactor vacuum vessel assembly robot, Fusion Engineering and Design 154 (2020) 111532. https://doi.org/10.1016/j.fusengdes.2020.111532

[8] M. Kanetomo, H. Kashima, T. Suzuki, Wafer-transfer robot for use in ultrahigh vacuum, Journal of Vacuum Science and Technology A: Vacuum, Surfaces, and Films 15(3) (1997) 1385-1388. https://doi.org/10.1116/1.580546

[9] M. Kanetomo, H. Kashima, T. Suzuki, Robot for use in ultrahigh vacuum, Solid State Technology 40(8) (1997) 63-68.

[10] H.C. Chen, S.M. Huang, Design and analysis of the power drive module for ultrahigh vacuum wafer-transfer robot, Journal of Energy and Power Engineering 4(6) (2010) 55-59.

[11] M. Cong, T. Li, Design and application of magnetic coupling used for ultra-high vacuum robot, International Journal of Intelligent Systems Technologies and Applications 8(1-4) (2010) 231-246. https://doi.org/10.1504/IJISTA.2010.030202

[12] M.J. Chung, S.J. Lee, Development of automatic wafer centering system for vacuum transfer robot using for semiconductor manufacturing, Applied Mechanics and Materials 607 (2014) 782785. https://doi.org/10.4028/www.scientific.net/AMM.607.782

[13] M. Cong, H. Wen, Y. Du, P. Dai, Coaxial twin-shaft magnetic fluid seals applied in vacuum wafer-handling robot, Chinese Journal of Mechanical Engineering 25(4) (2012) 706-714. https://doi.org/10.3901/CJME.2012.04.706

[14] L. Wang, S. Xu, W. Zhang, Y. Liu, Y. Xia, A cable-driven robot arm for visual tracking in tokamak vacuum vessel, IEEE International Conference on Robotics and Biomimetics (ROBIO2018) (2018) 1125-1131. https://doi.org/10.1109/ROBIO.2018.8665129

[15] O.V. Gradov, M.A. Gradova, A.A. Olkhov, A.L. Iordanskii, Charge propagation along the polymer fiber of polyhydroxybutyrate: Is it possible to apply the cable model? Key Engineering Materials 869 (2020) 246-258. https://doi.org/10.4028/www.scientific.net/KEM.869.246

[16] N. Zaporozhets, Application of air microejector in vacuum gripping device of industrial robot., Mekhanizatsiya i Avtomatizatsiya Proizvodstva (Moscow, USSR) 12 (1986) 25.

[17] S. Belinski, W. Trento, R. Imani-Shikhabadi, S. Hackwood, Robot design for a vacuum environment, Proceedings of the WSTL, 1 (1987) 95-103.

[18] M. Shirazi, Development and testing of a vacuum-compatible robot, Manuf. Rev. 1(4) (1988) 259-264.

[19] J. McCrea, J.T. Cerri, C.R. Hartsfield, Design of a zero-gravity, vacuum-based 3D printer robot for use of in-space satellite assembly, 2018 AIAA Aerospace Sciences Meeting (2018) 2201. https://doi.org/10.2514/6.2018-2201

[20] E. Grossman, I. Gouzman, Space environment effects on polymers in low earth orbit, Nuclear Instruments and Methods in Physics Research Section B: Beam Interactions with Materials and Atoms 208 (2003) 48-57. https://doi.org/10.1016/S0168-583X(03)00640-2

[21] B. Basu, S. Biswas, S. Dey, A. Maulik, A. Mazumdar, S. Raha, S. Saha, S. K. Saha, and D. Syam, Polyethylene terephthalate polymers at mountain altitude as cosmic ray heavy particle 
detector, Radiation Measurements 43 (2008) S262-S265.

https://doi.org/10.1016/j.radmeas.2008.04.021

[22] U.H. Hossain, W. Ensinger, Experimental simulation of radiation damage of polymers in space applications by cosmic-ray-type high energy heavy ions and the resulting changes in optical properties, Nuclear Instruments and Methods in Physics Research Section B: Beam Interactions with Materials and Atoms 365 (2015) 230-234. https://doi.org/10.1016/j.nimb.2015.06.007

[23] D. Bója, A. Major, The influence of cosmic radiation on the thermal properties of different polymers, Gradus 4(2) (2017) 351-356.

[24] A.A. Major, D. Boja, What can cosmic radiation cause in polymers? IOP Conference Series: Materials Science and Engineering 448(1) (2018) 012057. https://doi.org/10.1088/1757899X/448/1/012057

[25] D.I. Park, C.H. Park, Y. Yoo, Vibration simulation of hybrid type substrate handling robot in the vacuum environment, 12th International Conference on Control, Automation and Systems (2012) 2131-2134).

[26] A.C. Clarke, An optimum strategy for interstellar robot probes, Correspondence in Journal of the British Interplanetary Society 31 (1978) 438.

[27] G.L. Matloff, Robosloth: A slow interstellar thin-film robot, Journal of the British Interplanetary Society 49(1) (1996) 33-36.

[28] R. Noble, M.V. Sykes, Small body exploration technologies as precursors for interstellar robotics, Journal of the British Interplanetary Society 66 (2013) 15-24.

[29] E. Rhodes, A.J. Mauceri, M.M. Clarke, T.S. Lindsay, Paper Session IC-Robotics for Interstellar Missions, The Space Congress Proceedings 7 (1993) 35-41.

[30] D. Clery, Robot detector to map cosmos for clues to dark energy's force, Science 365(6458) (2019) 1066. https://doi.org/10.1126/science.365.6458.1066

[31] S. Vernov, B. Tverskoi, V. Yakovlev, E.V. Gorchakov, P. Ignatev, G. Lyubimov, N.V. Peresleg, O.A. Marchenko, T.E. Shvidkov, N. Kontor T. Morozova, Cosmic-ray measurements by Mars-2 robot space station, Izvestiya Akademii Nauk SSSR Seriya Fizicheskaya 38(9) (1974) 1859-1862.

[32] V.I. Gouliaev, T.V. Zavrazhina, Dynamics of a flexible multi-link cosmic robotmanipulator, Journal of Sound and Vibration 243(4) (2001) 641-657.

https://doi.org/10.1006/jsvi.2000.3409

[33] A. Ellery, Environment-robot interaction-the basis for mobility in planetary micro-rovers, Robotics and Autonomous Systems 51(1) (2005) 29-39.

https://doi.org/10.1016/j.robot.2004.08.007

[34] H. Omori, H. Kitamoto, A. Mizushina, T. Nakamura, T. Kubota, Satellite, planetary or terrestrial subsurface explorer robot based on earthworm locomotion, Journal of Robotics and Mechatronics 26(5) (2014) 660-661. https://doi.org/10.20965/jrm.2014.p0660

[35] L. Bai, W.J. Ge, X.H. Chen, M. Zhang, Research on hopping robot for planetary exploration, Robot, 31(4) (2009) 311-319.

[36] L. Bai, W. Ge, X. Chen, R. Chen, Design and dynamics analysis of a bio-inspired intermittent hopping robot for planetary surface exploration, International Journal of Advanced Robotic Systems 9(4) (2012) 109. https://doi.org/10.5772/51930 
[37] L. Bai, W. Ge, X. Chen, X. Kou, Design and implementation of a bio-inspired intermittent hopping robot for planetary surface exploration, Robot 34(1) (2012) 32-37.

https://doi.org/10.3724/SPJ.1218.2012.00032

[38] A.A. Pankine, K.M. Aaron, M.K. Heun, K.T. Nock, R.S. Schlaifer, C.J. Wyszkowski, A.P. Ingersoll, R.D. Lorenz, Directed aerial robot explorers for planetary exploration, Advances in Space Research 33(10) (2004) 1825-1830. https://doi.org/10.1016/j.asr.2003.07.049

[39] R. Bertrand, B. Schaefer, M. Van Winnendael, R. Rieder, European tracked micro-robot for planetary surface exploration, IFAC Proceedings Volumes 31(33) (1998) 37-44. https://doi.org/10.1016/S1474-6670(17)38384-2

[40] H. Das, X. Bao, Y. Bar-Cohen, R. Bonitz, R.A. Lindemann, M. Maimone, I.A. Nesnas, C.J. Voorhees, Robot manipulator technologies for planetary exploration, Smart Structures and Materials 1999: Smart Structures and Integrated Systems 3668 (1999) 175-182. https://doi.org/10.1117/12.350698

[41] A. Seeni, B. Schäfer, G. Hirzinger, Robot mobility systems for planetary surface explorationstate-of-the-art and future outlook: a literature survey, Aerospace Technologies Advancements 492 (2010) 189-208. https://doi.org/10.5772/6930

[42] I. Nanyageev, I. Shardyko, I. Dalyaev, Motion specification algorithms for both platform and arms of a mobile robot for planetary research, IOP Conference Series: Materials Science and Engineering 747(1) (2020) 012090. https://doi.org/10.1088/1757-899X/747/1/012090

[43] M. Maurette, M. Mellini, J. Silen, I. Tabacco, A. Morbidelli, R. Chatila, Meteorite at dome C? A project for the automated search for meteorites with a planetary exploration robot equipped with two radars, Meteoritics and Planetary Science Supplement 33 (1998) A100.

[44] R. Bertrand, B. Schaefer, R. Reider, European tracked micro-robot for planetary surface exploration, Space Technology 20(2) (2000) 55-64.

[45] G. Musser, Planetary science-NASA's robot rover scouts unknown terrain on the Angry Red Planet, Scientific American 290(3) (2004) 52-57. https://doi.org/10.1038/scientificamerican030452

[46] P.J. Westwick, Planetary exploration in extremis: JPL's robot explorers are the pride of NASA, but the lab nearly got shut down in the budget-cutting early'80s, Engineering and Science 69(4) (2006) 32.

[47] K. Ui, K. Nakaya, K. Konoue, H. Sawada, S. Tsurumi, M. Mori, R. Hodoshima, N. Maeda, H. Okada, N. Miyashita, M. Iai, O. Mori, S. Matunaga, Titech CanSat Project 2000: Report of Suborbital Flight and Balloon Experiment, in: M. Rycroft, N. Crosby (Eds.), Smaller Satellites: Bigger Business? Space Studies, 6, Springer, Dordrecht, 2000, pp. 417-418. https://doi.org/10.1007/97894-017-3008-2_62

[48] N. Sako, Y. Tsuda, S. Ota, T. Eishima, T. Yamamoto, I. Ikeda, H. Ii, H. Yamamoto, H. Tanaka, A. Tanaka, S. Nakasuka, CanSaT suborbital launch experiment-university educational space program using can sized pico-satellite, Acta Astronautica 48(5-12) (2001) 767-776. https://doi.org/10.1016/S0094-5765(01)00039-X

[49] T. Eishima, Y. Nakamura, S. Nakasuka, Space outreach program using CanSat-kit, Transactions of the Japan Society for Aeronautical and Space Sciences, Space Technology 7(26) (2009) 19-23. https://doi.org/10.2322/tstj.7.Tu_19 
[50] S.H. Won, H.Y. Jun, S.H. Kim, S.R. Lee, Very small satellite program for expending the space technology base: CanSat competition, Journal of the Korean Society for Aeronautical and Space Sciences 40(7) (2012) 636-645. https://doi.org/10.5139/JKSAS.2012.40.7.636

[51] R. Kawashima, CanSat leader training program: Past, present and future, Ciencia UANL 19(81) (2016) 76-82.

[52] A. Colin, B. Bermudez Reyes, G.E. Morrobel, G.A. Lira Ibarra, D.M. Zúñiga Rosales, L.A. Avalos de la Cruz, M. Villarreal Méndez, J. Mendoza Martínez, B. Alvarez Arce, Construcción de un picosatélite CanSat, Ciencia UANL 19(81) (2016) 34-38.

[53] A. Colin, A pico-satellite assembled and tested during the 6th CanSat Leader Training Program, Journal of Applied Research and Technology 15(1) (2017) 83-91. https://doi.org/10.1016/j.jart.2016.10.003

[54] L.A., Anchino, A.F., Torti, E.M., Dovis, E. Bernardi, R. Podadera, Implementacion de una Plataforma de Desarrollo CanSat Multiproposito, Elektron 3(2) (2019) 120-127.

https://doi.org/10.37537/rev.elektron.3.2.93.2019

[55] H.-U. Oh, H.-I. Kim, J.-K. Kim, J.-S. Choi, S.-H. Kim, Smartphone CanSat for actualization of real-time streaming video calls using remote screen touch system with shape memory alloy actuator, Transactions of the Japan Society for Aeronautical and Space Sciences 62(5) (2019) 256264. https://doi.org/10.2322/tjsass.62.256

[56] J. Nakaya, T. Takada, Y. Kajimura, H. Tsuchiya, N. Uezono, Y. Sasaoka, S. Ueta, M. Wakabayashi, K. Kitamura, Development of CubeSat Ground Model Extended from CanSat: Application to Space Education at KOSEN, Transactions of the Japan Society for Aeronautical and Space Sciences, Aerospace Technology Japan 18(5) (2020) 281-287.

https://doi.org/10.2322/tastj.18.281

[57] J.S. Rodríguez, A.Y. Botero, D.V. Lopera, J.G. Serna, F. Botero, Experimental approach for the evaluation of the performance of a satellite module in the CanSat form factor for in situ monitoring and remote sensing applications, International Journal of Aerospace Engineering 2021(81) (2021) 1-28. https://doi.org/10.1155/2021/8868797 